\title{
Three-Dimensional Culture of Umbilical Cord Mesenchymal Stem Cells Effectively Promotes Platelet Recovery in Immune Thrombocytopenia
}

\author{
Xiangcui Gong, ${ }^{a, b}$ Di Sun, ${ }^{c}$ Zhenghao Li, ${ }^{d}$ Qing Shi, ${ }^{a}$ Dong Li,${ }^{a}$ and Xiuli Ju*a \\ ${ }^{a}$ Department of Pediatrics, Qilu Hospital of Shandong University; Jinan 250012, P. R. China: ${ }^{b}$ Department of \\ Pediatrics, Qingdao Women and Children's Hospital; Qingdao 266034, P. R. China: ${ }^{c}$ Yantai Central Blood Station; \\ Yantai, 264003, P. R. China: and ${ }^{d}$ Department of Pediatrics, Yidu Central Hospital of Weifang; Qingzhou 262500, \\ P. R. China.
}

Received December 8, 2019; accepted March 30, 2020; advance publication released online April 21, 2020

\begin{abstract}
Mesenchymal stem cells (MSCs) can effectively regulate immune cell functions and therefore are promising for the treatment of autoimmune disorders, such as immune thrombocytopenia (ITP). Recent research has shown that three-dimensional (3D) culture method have many advantages over conventional culture with respect to MSC secretion and immunogenicity. In this study, 2D and 3D cultured MSCs were used to evaluate cytokine secretion, extracellular matrix (ECM) gene expression, immune regulatory activity, and therapeutic effects in a mouse model of ITP. MSCs cultured on scaffolds had higher expression levels of immune regulatory genes, such as IDO1, $H L A-G$, and $P T G S 2$, and greater inhibitory activity against lymphocyte activation that those of 2D-MSCs. In addition, 3D-MSCs exhibited higher ECM expression and greater protection against interferon- $\gamma($ IFN- $\gamma)$-induced apoptosis. In a mouse study, ITP was induced by guinea pig anti-mouse platelet serum injections. Based on enzyme-linked immunosorbent assays, serum levels of the suppressive cytokine interleukin (IL)-10 were higher and IFN- $\gamma$ levels were lower after intravenous injection with 3D-MSCs and with 2D-MSCs. Additionally, 3D-MSCs improved the body weight, spleen index, and platelet index relative to those for $2 \mathrm{D}$-MSCs. Bone marrow homing was also significantly enhanced in the 3D group. Therefore, the 3D culture of MSCs is an effective technique for the treatment of ITP.
\end{abstract}

Key words immune thrombocytopenia; immunoregulation; umbilical cord mesenchymal stem cell; threedimensional culture

\section{INTRODUCTION}

Immune thrombocytopenia (ITP) is an autoimmune disorder characterized by both reduced platelet counts and the suppression of megakaryocytes. Abnormalities of cell-mediated immunity are known to contribute to the pathological process. Like many autoimmune diseases, ITP has a $\mathrm{T}$ helper (Th) cell type 1 bias and a reduced activity of T-regulatory cells. ${ }^{1)}$ Mesenchymal stem cells (MSCs) show immunomodulatory properties and have been utilized extensively for the treatment of autoimmune diseases. The impaired immunosuppressive capacity of MSCs in patients with ITP might contribute to disease development., ${ }^{2,3)}$ Based on in vitro cytological experiments, MSCs significantly inhibit interleukin (IL)-2 and interferon- $\gamma($ IFN $-\gamma)$ secretion by Th1 cells and promote the release of IL- 4 and IL-10 by Th2 cells in ITP; this regulates the balance between Th1 and Th2 activity and up-regulates $\mathrm{CD}^{+} \mathrm{CD} 25^{+} \mathrm{T}$ cells, inducing immunologic tolerance in ITP. ${ }^{4)}$ Accordingly, MSCs could improve platelet counts in mice with ITP.

A small number of clinical trials have shown that MSC transfusions are effective for the treatment of ITP. ${ }^{5)}$ MSCs immunosuppress IL-22 in patients with ITP via soluble cellular factors. Moreover, MSCs inhibit the proliferation of activated $\mathrm{CD}^{+}{ }^{\mathrm{T}}$ cells. $\left.{ }^{6}\right)$ The immunomodulatory function of MSCs is positively correlated with the dose; however, high-dose MSCs are associated with an increase in adverse reactions, such as increased acute pulmonary embolism. ${ }^{7)}$ Therefore, it is essential to improve immune regulation by individual MSCs.

The advantages of three-dimensional (3D) cell culture are increasingly recognized by cell biologists. For example, 3D culture can increase the expression of the pluripotency gene $N A N O G$ in MSCs by relaxation of cytoskeleton tension. ${ }^{8)}$ Under 3D conditions, there are multi-directional cell-matrix interactions. ${ }^{9)}$ The spatial conformation of 3D scaffolds can promote interactions between cells and enhance the secretory function of MSCs. ${ }^{10)}$ Furthermore, 3D cultured MSCs exhibit lower immunogenicity and higher immunosuppressive capacity than those of 2D cultured MSCs. ${ }^{11)}$ These results suggest that MSCs cultured in 3D conditions may have beneficial effects in immune-related cell therapy. We speculate that ITP can be treated more effectively using 3D MSCs than using 2D MSCs. To evaluate this hypothesis, we prepared mouse platelet antigen for guinea pig immunization and used it to construct a chronic ITP mouse model by serum injection, resulting in a persistent reduction in platelets. Finally, the effect of MSCs was evaluated.

\section{MATERIALS AND METHODS}

Culture of Human Umbilical Cord Mesenchymal Stem Cells (UC-MSCs) Human umbilical cords were obtained from normal pregnancies in the Department of Obstetrics of Qilu Hospital. The use of human umbilical cords was approved by the Ethics Committee of Qilu Hospital, Shandong University (QL-TXB-2019004), and written informed consent was obtained from all participants. UC-MSCs were cultured according to our previously described methods. ${ }^{12,13)}$ Briefly, UC-MSCs were cultured in Dulbecco's modified Eagle's medium (DMEM)/F-12 (Gibco; Thermo Fisher Scientific Inc., 
Waltham, MA, U.S.A.) supplemented with $10 \%$ fetal bovine serum (FBS; Gibco), $100 \mathrm{U} / \mathrm{mL}$ penicillin (Gibco), and $100 \mathrm{~g} / \mathrm{mL}$ streptomycin (Gibco) in a fully humidified atmosphere with $5 \% \mathrm{CO}_{2}$ and $21 \% \mathrm{O}_{2}$ at $37^{\circ} \mathrm{C}$. UC-MSCs between passages 4 and 6 were used for all analyses.

Three-Dimensional Culture of UC-MSCs The polyvinyl fluoride (PVF) 3D culture scaffold was purchased from Bio-carrier Biotech (Binzhou, China). Prior to cell seeding, scaffolds were pre-incubated with phosphate buffer for $2 \mathrm{~h}$. Adherent UC-MSCs were trypsinized and resuspended in media at $5 \times 10^{6}$ cells $/ \mathrm{mL}$. Each $10 \times 10 \times 2 \mathrm{~mm}$ scaffold was seeded in a 12 -well plate with $500 \mu \mathrm{L} /$ well cell suspension for $1 \mathrm{~h}$ at $37^{\circ} \mathrm{C}$ before additional medium $(500 \mu \mathrm{L} /$ well) was added. The medium was changed every day. Conventional 2D culture with tissue culture plastic served as a control. After $48 \mathrm{~h}$ of culture, for both 3D and 2D conditions, the cells were digested with trypsin and ethylenediaminetetraacetic acid (EDTA) and then washed for further phenotypic analyses. UC-MSCs cultured with 3D scaffolds were observed by 4',6-diamidino-2-phenylindole (DAPI; Abcam, Cambridge, U.K.) staining and fluorescence microscopy (IX71; Olympus, Tokyo, Japan). Some 3D cultured cells were fixed with glutaraldehyde and used for scanning electron microscopy (SEM; Sigma300, Zeiss, Oberkochen, Germany). Fluorescein isothiocyanate (FITC) Phalloidin (working concentration $5 \mu \mathrm{g} / \mathrm{mL}$, BMD00082; AmyJet Scientific Inc., Wuhan, China) was used to stain the cytoskeletons of 2D and 3D MSCs.

The following formula was used to estimate cell growth: population doubling time $(\mathrm{PDT})=t \lg 2 /(\lg \mathrm{NH}-\operatorname{lgNI})$, where $t$ represents the culture duration $(\mathrm{h}), \mathrm{NH}$ is the harvested cell count, and NI is the inoculum cell count. ${ }^{14)}$

Phenotyping of UC-MSCs Flow cytometry was performed to characterize UC-MSCs cultured in 2D or 3D environments. The following cell surface epitopes were detected: PE-conjugated anti-human CD29 (4303570; eBioscience, San Diego, CA, U.S.A.), CD31 (4303600; eBioscience), CD34 (4302805; eBioscience), CD44 (4330029; eBioscience), CD45 (4343394; eBioscience), CD73 (4344363; eBioscience), CD90 (4307303; eBioscience), and CD105 (1983608; eBioscience). They were compared with corresponding isotype control antibodies. One million cells were stained with $10 \mu \mathrm{L}$ of each antibody in $100 \mu \mathrm{L}$ of phosphate-buffered saline (PBS) for $15 \mathrm{~min}$ at $25^{\circ} \mathrm{C}$. Flow cytometry was performed using the Guava easyCyte $6 \mathrm{HT}$ (EMD Millipore, Billerica, MA, U.S.A.), and data were examined using Guava Incyte (version 2.8, EMD Millipore).

Real-Time Quantitative PCR (qPCR) Cells were counted and equal numbers were used for nucleic acid extraction. Total RNA was obtained from each group of UC-MSCs using TRIzol (Invitrogen, Carlsbad, CA, U.S.A.) according to the manufacturer's protocol. After balancing the nucleic acid concentration of the two groups, qRT-PCR was performed to analyze the expression levels of various immunoregulationrelated genes and ECM-related genes (see Supplementary Table 1 for primers). Total RNA was reverse-transcribed to cDNA using the Reverse Transcription Kit (Beyotime, Shanghai, China) according to the manufacturer's instructions. qRTPCR was conducted using an ABI 7500 PCR system (Applied Biosystems, Foster City, CA, U.S.A.) and SYBR Green I Dye (Toyobo, Osaka, Japan). All other reagents and primers were obtained from Boshang Biotech. Co., Ltd. (Jinan, China). Glyceraldehyde-3-phosphate dehydrogenase $(G A P D H)$ was used as an internal control. The expression of each gene was determined using the $2^{-\triangle \Delta C T}$ method. The qPCR conditions were as follows: $95^{\circ} \mathrm{C}$ for $4 \mathrm{~min}, 94^{\circ} \mathrm{C}$ for $15 \mathrm{~s}$, and $60^{\circ} \mathrm{C}$ for $1 \mathrm{~min}$, for a total of 40 cycles. Data were analyzed using Sequence Detection Software 1.4 (Applied Biosystems). Data are reported as means \pm standard deviation (S.D.) of at least three independent experiments. mRNA expression levels are presented as fold changes relative to $2 \mathrm{D}$ culture groups.

Inhibition of Lymphocyte Proliferation Human umbilical cord blood samples $(n=6 ; 3$ males and 3 females) were obtained from mothers undergoing full-term deliveries at the Department of Obstetrics at Qilu Hospital of Shandong University (Jinan, China) after informed written consent was obtained. The use of umbilical cord blood was approved by the Ethics Committee of Shandong University Qilu Hospital (QL-TXB-2019004). Human cord blood mononuclear cells (CB-MNC) were isolated and collected by lymphocyte separation medium (LTS1077; TBD, Tianjin, China) and suspended in DMEM/F12 (Hyclone, Logan, UT, U.S.A.) containing 10\% FBS and $2 \mathrm{mM} / \mathrm{mL}$ glutamine. After the addition of an antiCD3 monoclonal antibody (Novoprotein, Shanghai, China; final concentration $100 \mathrm{ng} / \mathrm{mL}$ ) and IL-2 (Novoprotein; final concentration $10 \mathrm{ng} / \mathrm{mL}$ ), MNCs were incubated at $100 \%$ humidity, $37^{\circ} \mathrm{C}$ and $5 \% \mathrm{CO}_{2}$. One day later, activated lymphocytes were used for the inhibition test.

For the 2D-MSC group, MSCs were cultured on a 96-well plate (Corning, Inc., Corning, NY, U.S.A.) at $2 \times 10^{4}$ cells and $200 \mu \mathrm{L} /$ well, with 12 duplicate wells for each group. After $1 \mathrm{~h}$, when the cells completely adhered to the wall, mitomycin-C (MMC, $10 \mu \mathrm{g} / \mathrm{mL}$; Sigma, St. Louis, MO, U.S.A.) was added to the medium and incubated for $3 \mathrm{~h}$ at $37^{\circ} \mathrm{C}$. Medium containing MMC was discarded and the cells were then washed with normal medium 6 times. For the 3D-MSC group, the PVF $3 \mathrm{D}$ scaffold was placed in a 96-well plate, with $2 \times 10^{4}$ cells and $100 \mu \mathrm{L} /$ well. After the cells attached to the wall for $1 \mathrm{~h}$, $100 \mu \mathrm{L}$ of MMC-containing medium was added to obtain a final concentration of $10 \mu \mathrm{g} / \mathrm{mL}$. Cells were then washed with normal medium 6 times after $3 \mathrm{~h}$ and supplemented with ordinary medium. For co-culture, the cell count ratio of MSCs to lymphocytes was adjusted to $1: 10,1: 20$, or $1: 40$ by the addition of activated lymphocytes. The control group contained only activated lymphocytes. The 96-well plate was incubated at $37^{\circ} \mathrm{C}$ and a $5 \% \mathrm{CO}_{2}$ for $3 \mathrm{~d}$. Then, the lymphocytes were washed and added to a new plate, followed by counting by the CCK-8 (Beyotime) colorimetric method. Lymphocyte counts were determined by measuring the optical density (OD) at $450 \mathrm{~nm}$. The rate of inhibition of lymphocyte proliferation by MSCs was calculated based on cell counts according to the formula: inhibition rate $=($ control group - experimental group $) /$ control group.

Anti-inflammatory Stress Test Both 2D-MSCs and 3DMSCs were exposure to IFN- $\gamma$ (R\&D Systems, Minneapolis, $\mathrm{MN}$, U.S.A.) to induce inflammatory injury. Based on preliminary results, $50 \mathrm{ng} / \mathrm{mL}$ IFN- $\gamma$ for $12 \mathrm{~h}$ was selected to produce reversible damage. 2D-MSCs not treated with inflammatory factors were used as the control group.

Construction of the ITP Mouse Model and Stem Cell Therapy Female BALB/c mice $(n=30$, weighing $20 \pm 2 \mathrm{~g}$; 8 weeks old) and guinea pigs (male, weighing $250 \pm 20 \mathrm{~g} ; 8$ weeks old) were purchased from Beijing Charles River Experimental Animal Technical Co., Ltd. Animal studies were 


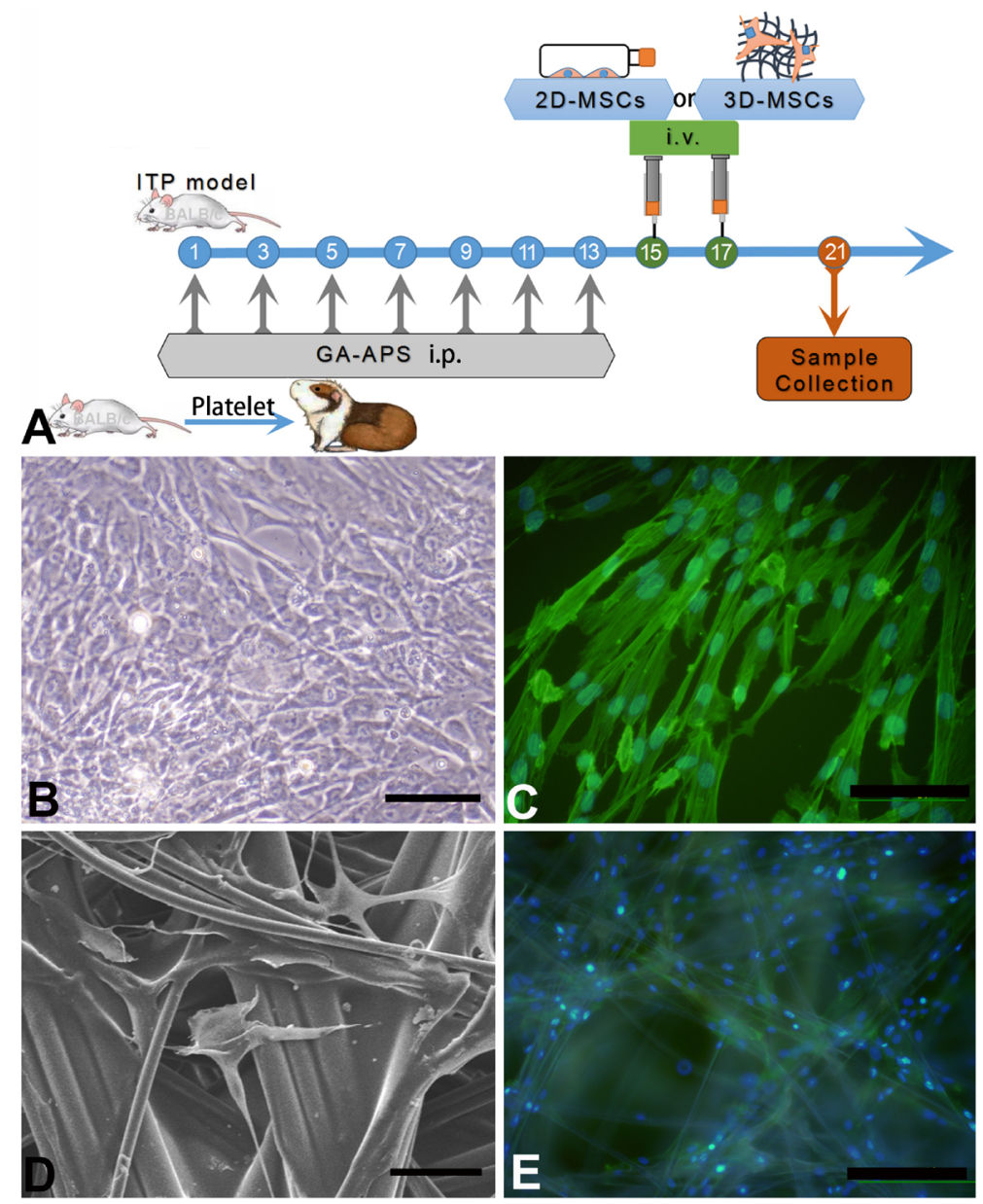

Fig. 1. Experimental Design and Cell Morphology

(A) Schematic diagram of the experimental flow. (B) 2D culture of UC-MSCs under a light microscope. Bar $=200 \mu \mathrm{m}$. (C) Observation of the cytoskeleton and nucleus of UC-MSCs in 2-D culture by fluorescence microscopy. Bar $=200 \mu \mathrm{m}$. (D) Observation of cell adhesion to a polystyrene scaffold in 3D culture by S.E.M. Bar $=100 \mu \mathrm{m}$. (E) Observation of the cytoskeleton and nucleus of UC-MSCs in 3-D culture. Bar $=200 \mu \mathrm{m}$. (Color figure can be accessed in the online version.)

approved by the Ethics Committee for Animal Experiments of Qilu Hospital, Shandong University. Forty BALB/c mice were randomly divided into four groups: normal group (Nor), model group (Mod), 2D-MSC Therapy Group (2D), and 3D-MSC Therapy Group (3D), with 10 mice per group. To prepare the guinea pig anti-mouse platelet serum (GP-APS), the platelets in the venous blood of BALB/c mice were obtained and immunized to guinea pigs. An ITP mouse model was established by the intraperitoneal injection of GP-APS (dilution 1:4) at $10 \mu \mathrm{L} / \mathrm{g}$. Antiserum was injected on days $1,3,5,7,9,11$, and 13 to maintain low platelet levels. Platelets were counted every $3 \mathrm{~d}$ and the criterion for successful modeling was the maintenance of platelets at $11-25 \%$ of the original level (Animal Automatic Hematology Analyzer, BC-2800vet; Mindary, Shenzhen, China). General conditions of animals were observed and the spleen/body mass index was recorded. For the $2 \mathrm{D}$ and 3D groups, 2D-MSCs or 3D-MSCs at $2 \times 10^{6} / 200 \mu \mathrm{L}$ per mouse were transfused from the caudal vein for treatment on days 15 and 17. On day 21, all mice were sacrificed to measure the spleen index and platelet concentration. IL-10, and IFN- $\gamma$ levels in the peripheral blood serum were quantified using an enzyme-linked immunosorbent assay (ELISA) kit according to the manufacturer's instructions (R\&D Systems). The specific experimental design is summarized in Fig. 1A.

Homing and Survival Time of MSCs in Vivo Female
BALB/c mice $(20 \pm 2 \mathrm{~g})$ were randomly divided into 2D-MSC and 3D-MSC groups, with 6 mice in each group. The UCMSCs from male infants were then intravenously injected with $2 \times 10^{6} / 200 \mu \mathrm{L}$ per mouse. At $6,12,24,48$, and $72 \mathrm{~h}$ post injection, three $\mathrm{BALB} / \mathrm{c}$ mice were sacrificed at each time point, and bone marrow, lung, liver, spleen, and kidney tissue samples were collected for DNA amplification. The Genomic DNA Extraction Kit (Beyotime) was used to isolate the total genomic DNA from different tissues following the manufacturer's instructions. Then, human Sex-determining Region $\mathrm{Y}$ (SRY) and mouse ACTIN in different tissues were detected by qPCR (see Supplementary Table 1 for primers). The SRY value in the liver for each mouse was set to 1 .

Statistical Analysis All data are presented as means \pm standard error of the mean (S.E.M.) of three separate experiments. Data were analyzed by the Student's $t$-test for comparison between two groups or ANOVA for comparisons among multiple groups, as appropriate. Differences were considered statistically significant at $p<0.05$. Statistical analyses were performed using SPSS Statistics 17.0 (Version X; IBM, Armonk, NY, U.S.A.).

\section{RESULTS}

Characteristics of MSCs Cultured in 3D MSC cells in 

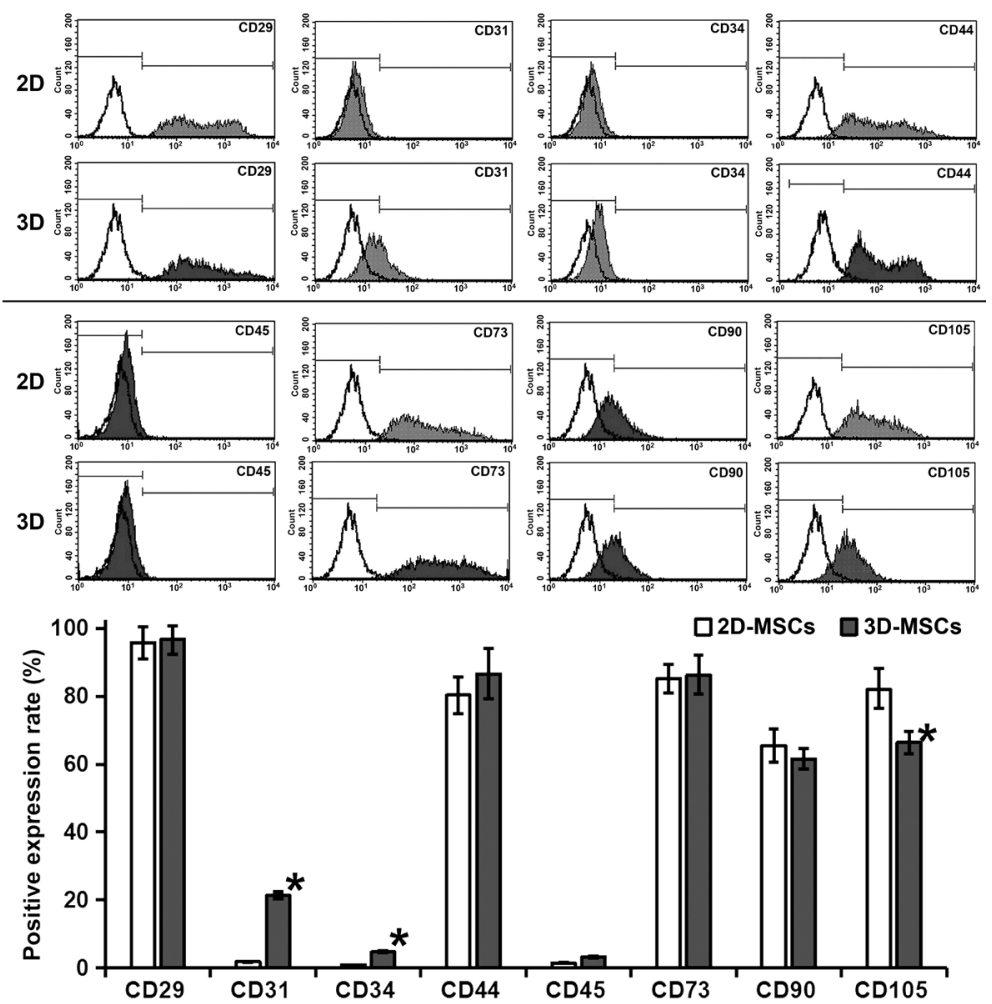

口2D-MSCs Q3D-MSCs

Fig. 2. Effect of 3D Culture on the Phenotype of UC-MSCs

$* p<0.05$ vs. $2 \mathrm{D}$ group.

2D culture exhibited a typical fibroblast-like morphology and grew in a denser monolayer after reaching confluence (Figs. $1 \mathrm{~B}, \mathrm{C})$. The induction of differentiation is described previously. ${ }^{15,16)}$ However, when cells were cultured on polystyrene 3D scaffolds, the cell morphology became compact (Figs. 1D, E). UC-MSCs could adhere to polystyrene scaffolds well, but the rate of proliferation was slower than that of $2 \mathrm{D}$ culture. In the $2 \mathrm{D}$ culture system, the doubling time of UC-MSCs in the 4 th generation was about $32 \mathrm{~h}$, while in the $3 \mathrm{D}$ culture, the doubling time was extended to $42 \mathrm{~h}$.

A flow cytometry analysis of UC-MSCs revealed several key differences between groups. In particular, the rate of CD31- and CD34-positive cells was higher and the rates of CD105-positive cells were lower in 3D-MSCs than in 2DMSCs $(p<0.05$, Fig. 2).

Comparison of Immunosuppressive Properties of Human UC-MSCs in 2D and 3D Culture Systems In ITP, it is important to consider the immune regulatory properties of UC-MSCs. Accordingly, we used qRT-PCR to detect the expression of several genes closely related to immune regulation. All eight genes were more highly expressed after 3D culture than after 2D culture. Among them, HLA-G, IDOI, $P T G S 2$, and TGF- $\beta 1$ were significantly up-regulated $(p<0.05$, Fig. 3A). We further evaluated the inhibitory effect of UCMSCs on the proliferation of activated lymphocytes by mixed culture. UC-MSCs cultured in 3-D could inhibit the proliferation of activated lymphocytes more effective, regardless of the co-culture ratio ( $p<0.05$, Fig. 3B).

Increased Extracellular Matrix Production and Stress Resistance of MSCs Cultured in 3D Compared with the control group, UC-MSCs cultured in 3D expressed higher levels of collagen type I, fibronectin and integrin (Fig. 3C).
When MSC is infused back into ITP mice, it will first face the severe environmental test of inflammatory environment, especially inflammatory factors. So we evaluated whether 3D-culture could improve the ability of MSCs to resist inflammatory factors IFN- $\gamma$ through flow cytometry apoptotic kit. According to the results of the pre-experiment, we directly selected $50 \mathrm{ng} / \mathrm{mL}$ IFN $-\gamma$ to intervene MSC. Compared with 2D MSCs without inflammatory factors (control group), this concentration of IFN- $\gamma$ increased the proportion of apoptotic cells in the late stage $(p<0.05)$, but had little effect on the early stage of apoptosis. In the 3D group, IFN- $\gamma$ stimulation did not increase both of early and late apoptotic cells ratio (Figs. 3D, E).

MSCs Cultured in 3D Can Rapidly Restore Platelet Counts in an ITP Mouse Model We induced ITP in a murine model by daily injections with anti-platelet serum. After the first injection of GP-APS, the activity of mice in the ITP model group began to decrease, and this was accompanied by a decrease in food and water intake. In the ITP model group, agility of mice also decreased significantly, accompanied by loose stools and a reduced final weight $(p<0.05$, Fig. 4A). After the infusion of MSCs, the spleen index decreased significantly in 3D-MSC group $(p<0.05$, vs. Model group. Fig. 4B). No mice died during the experiment.

Twenty-four hours after GP-APS injection, the platelet count in ITP model mice decreased significantly and remained low throughout the 2 -week modeling period ( $p<0.05$ compared with the normal group, Fig. 4C). After two MSC treatments, the platelet counts in the two MSC groups increased significantly on day $21 \quad(p<0.01$ compared with the model group, Fig. 4D), particularly in the 3D-MSC group $(p<0.05$ vs. Model group, Fig. 4D), but there was no significance between the 3D-MSC and 2D-MSC groups. 

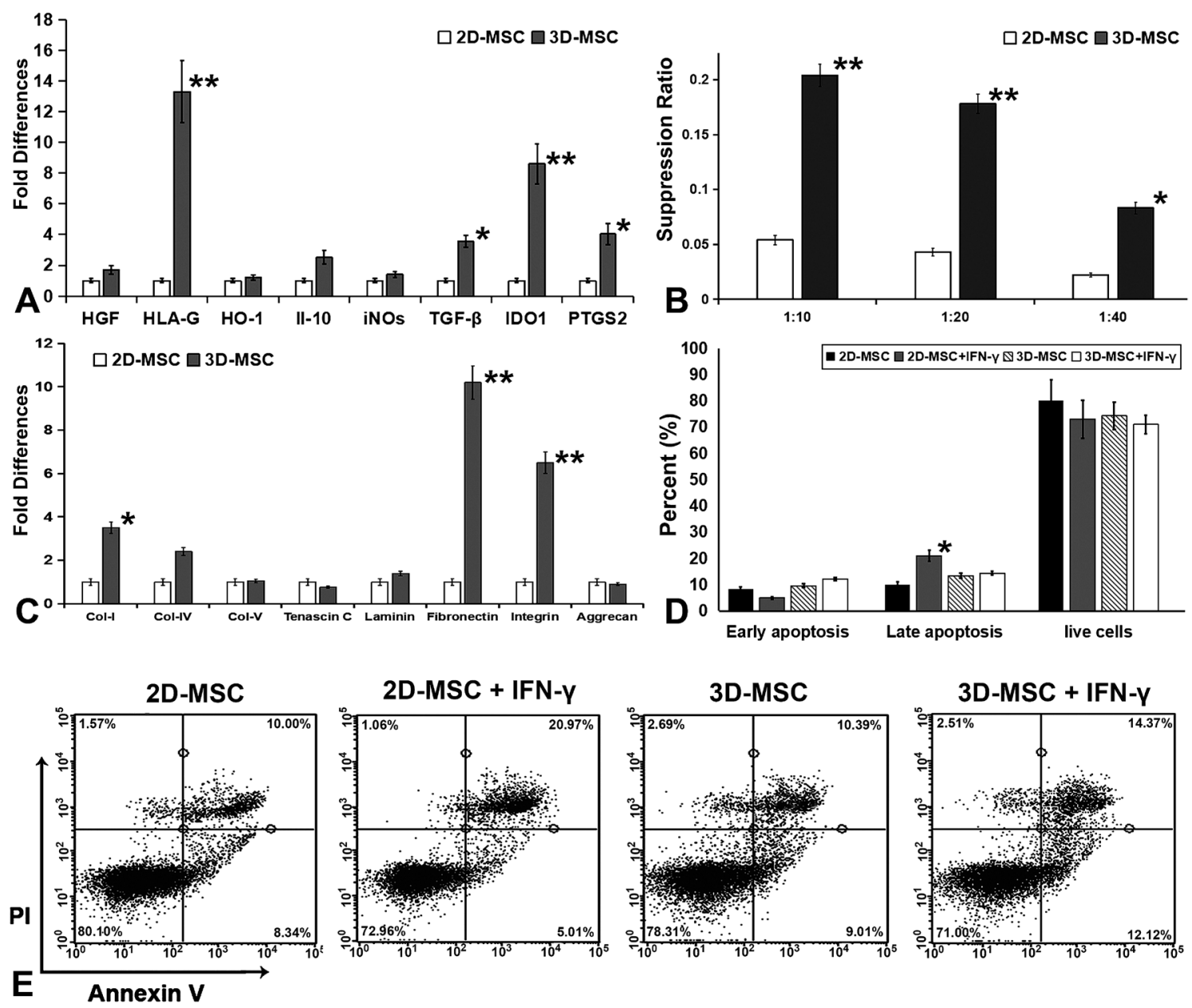

Fig. 3. 3D Culture Can Enhance Immune Regulation, Extracellular Matrix Expression, and Anti-inflammatory Activity of UC-MSCs

(A) qRT-PCR showed that $3 \mathrm{D}$ culture could significantly increase the expression of immune-related genes. ( $* p<0.05, * * p<0.01 v s .2 \mathrm{D}$ group.) (B) Co-culture results showed that UC-MSCs cultured in $3 \mathrm{D}$ inhibited the proliferation of activated lymphocytes more effectively than $2 \mathrm{D}$ culture. $(* p<0.05$, $* * p<0.01 v s$. $2 \mathrm{D}$ group.) $(\mathrm{C})$ Quantitative PCR showed that $3 \mathrm{D}$ culture could increase the expression of some extracellular matrix-related genes, especially fibronectin, integrin, and Col-I. ( $* 2<0.05$, $*^{*} p<0.01$ vs. 2D group.) (D, E) Although the inflammatory factor IFN- $\gamma$ can increase the proportion of apoptosis in 2D MSC, it does not increase the proportion of apoptosis in 3D MSC. $(* p<0.05$, vs. control group.)

To explore the anti-inflammatory effect of MSCs, we measured the levels of cytokines (IFN- $\gamma$ and IL-10) in the peripheral serum of mice. The serum levels of IL-10 were lower and IFN $-\gamma$ were higher in the ITP model group than in the normal group $(p<0.05$, Fig. 4E). After MSC treatment, the expression of IL-10 increased, while the expression of IFN- $\gamma$ decreased significantly compared with levels in the model group ( $p<0.05$, Fig. $4 \mathrm{E})$, particularly in the 3D-MSC group, but there were no significant differences between the 3D-MSC and 2D-MSC groups.

Three-Dimensional UC-MSCs Extended Survival in Vivo Using UC-MSCs from male infants for transfusion, we tracked whether cells could survive in various tissues of female mice by PCR detection of the male-specific $S R Y$ gene. At early time points, cells were mainly concentrated in the lungs, and there was no significant difference between the 3D-MSC group and the 2D-MSC group in the lungs. However, bone marrow migration was significantly higher in the 3D-MSC group than in the $2 \mathrm{D}$ group $(p<0.05$, Fig. 5$)$. At $72 \mathrm{~h}, S R Y$ was still detected in the bone marrow of the 3D-MSC group but not in the 2D-MSC group. Compared with the 2D-MSC group, the 3D-MSC group exhibited slight, delayed increases in the spleen and kidney, but the differences were not significant.

\section{DISCUSSION}

Our results proved that 3D MSCs are superior to 2D MSCs in terms of immune regulation and ECM secretion. In addition, UC MSCs in 3D culture could more effectively resist the effects of inflammatory factors, and with lower rates of apoptosis than those in $2 \mathrm{D}$ culture. In ITP model rats, improvements in the body weight, spleen index, and platelet index were better using 3D MSCs than using 2D MSCs. The ability of homing to bone marrow was also significantly enhanced in 3D group.

ITP is a common autoimmune blood disorder characterized by impaired platelet production, and platelet over-destruction is mediated by anti-platelet antibodies. ${ }^{15)}$ The passive ITP mouse model obtained by the transfer of anti-platelet antibodies has been utilized extensively for research. ${ }^{16)}$ This method can lead to substantial platelet destruction, which resembles the immunological mechanism underlying ITP, and will not affect peripheral blood leukocytes, erythrocytes, and other cells of mice. ${ }^{17)}$ Accordingly, we prepared anti-mouse platelet serum of guinea pigs to establish a chronic ITP mouse model. Antiserum was injected every other day to simulate sustained immune thrombocytopenia in mice. This method can rapidly 


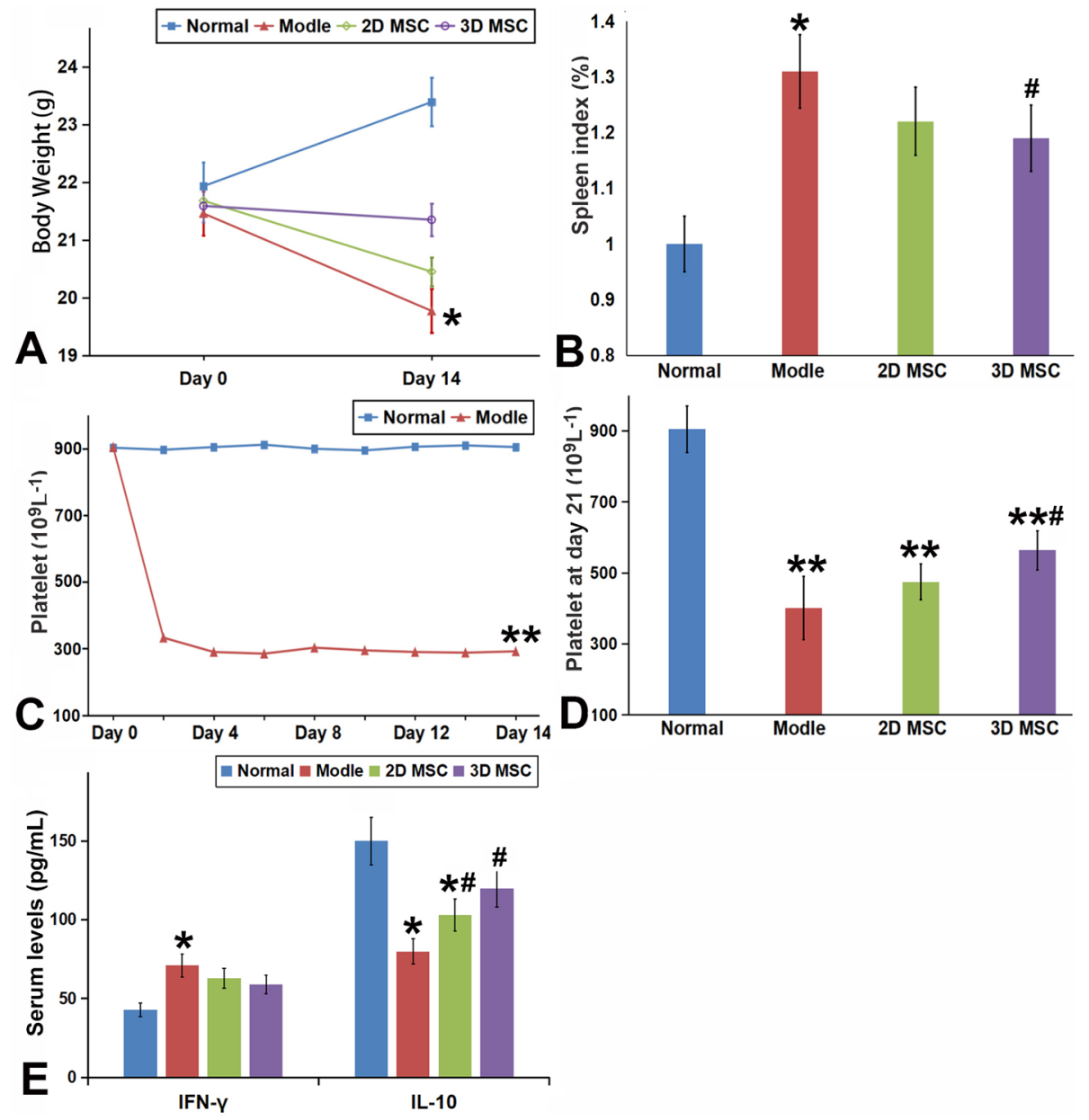

Fig. 4. Therapeutic Effect of 2D- or 3D-MSCs on ITP Mice

(A) Effects of ITP modeling and MSC treatment on the body weight of mice. (B) Changes in the spleen index changed during model establishment and treatment. Compared with the model group, the 3D MSC group had a significantly lower spleen index. (C) In the process of ITP modeling, the number of platelets decreased continuousl ; (D) Infusion of 2D MSCs and 3D MSCs can increase platelet counts, but the effect was greater in the 3D MSC group; (E) After ITP modeling, the concentration of IFN-r in the serum increased, while that of IL-10 decreased. Infusion of 2D MSCs and 3D MSCs can reverse this trend, especially in the 3D MSC group. $(* p<0.05 v s$. Normal group; ${ }^{* *} p<0.01 v s$. Normal group; $\# p<0.05 v s$. Model group). (Color figure can be accessed in the online version.)

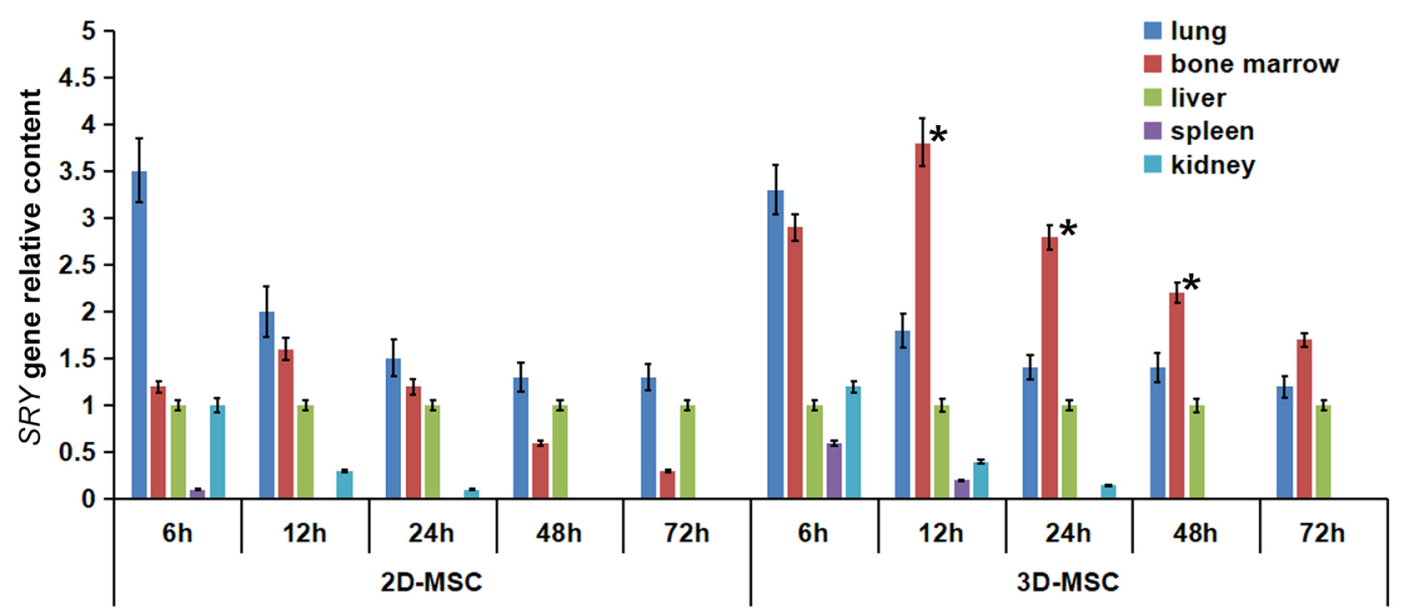

Fig. 5. In Vivo Survival Time Was Significantly Longer in the 3D-MSC Group than in the 2D-MSC Group

The value of $S R Y$ normalized to mouse $A C T I N$ and the lung index were set to 1 . Bone marrow migration was higher in the $3 \mathrm{D}-\mathrm{MSC}$ group than in the $2 \mathrm{D}$ group ( $* p<0.05$ compared to the 2D-MSC group, $n=6$ ). (Color figure can be accessed in the online version.)

reduce the number of platelets in mice, with low levels for least $14 \mathrm{~d}$ and a mortality of zero. Xenotransplantation methods afford an opportunity to study the effect of human MSCs in mice with ITP. In this study, we used human derived cells to treat mouse model. Although human MSCs get immune rejected rapidly in immune competent mice, many related cell therapy studies have confirmed that human MSCs can play a good therapeutic effect in rodents. ${ }^{18-21)}$ Some studies suggest 
that mouse MSCs are less effective than human MSCs in immune regulation. ${ }^{22)}$ In general, human cytokines have poor activities on other species, especially experimental rodents. But the species difference of extracellular matrix (ECM) is very small, we postulate that the secretion of ECM proteins like laminins by MSCs may create a niche that is beneficial for tissue repair and immune regulation. In addition to the phenotypical differences, murine and human MSCs differ in their mechanisms of immunosuppression. Mouse mesenchymal stem cells use nitric oxide (NO) to inhibit inflammatory response. $^{23,24)}$ Human MSCs suppresses $\mathrm{T}$ cell proliferation through indoleamine 2,3-dioxygenase (IDO), an enzyme that converts the essential amino acid tryptophan into the immunosuppressive catabolite, kynurenine. ${ }^{25)}$ This may be one of the reasons why human MSCs can play an immunosuppressive role in allogeneic animals.

In ITP, MSCs are mainly involved in immune regulation. MSCs can regulate immune cells via cell contact and secretion. The immunosuppressive factors expressed on the cell surface can directly inhibit immune cell activity, such as programmed death ligand 1 (PD-L1) and Fas ligand (Fas-L), leading to a loss of immune cell function. ${ }^{26,27)}$ This tight binding of MSCs to activated immune cells enhances immunosuppression. ${ }^{28)}$ The immunosuppressive cytokines secreted by MSCs mainly include transforming growth factor $\beta$ (TGF- $\beta$ ), hepatocyte growth factor (HGF), prostaglandin E2 (PGE2), and other anti-inflammatory factors to inhibit immune cell growth. ${ }^{29,30)}$ These factors can also have immunosuppressive effects by promoting regulatory $\mathrm{T}$ cells (Tregs) and macrophages. ${ }^{31,32)}$ Therefore, MSCs play a key role in maintaining immune homeostasis by interacting with cytokines, chemokines, and cell surface molecules. The proinflammatory cytokines IFN- $\gamma$, tumor necrosis factor (TNF)- $\alpha$, and IL-1B can effectively improve the immunosuppressive effect of MSCs. When these cytokines are used alone or in combination, they can stimulate the expression of several chemokines and inducible nitric oxide synthetase in MSCs. Chemokines cause T cells to migrate to the vicinity of MSCs, and nitric oxide inhibits $\mathrm{T}$ cell immune response. ${ }^{33,34)}$ However, pretreatment with inflammatory cytokines, such as IFN- $\gamma$, may also have side effects. Our results also show that IFN- $\gamma$ can lead to an increase in apoptosis. We believe that it may be possible to improve the immunoregulatory activity of MSCs by 3D culture and tissue engineering.

Our results show that $3 \mathrm{D}$ culture can effectively upregulate the expression of immune regulation-related genes in MSCs, such as IDO1, HLA-G, PTGS2, and other immune regulatory factors, which could better inhibit lymphocyte activation. The results of our lymphocyte inhibition test further supported the effectiveness of 3D cultured MSCs. Previous studies have reported that local transplantation of 3D aggregates can increase the expression of immunoregulatory factors and improve the local inflammatory environment of cells at the injection site. The increased expression of IDO promotes an increase in the proportion of M2 macrophages, thus reducing the inflammatory response. ${ }^{35)}$ One study demonstrated that 3D MSCs exert strong anti-inflammatory effects through upregulated TNFAIP6/TSG6. ${ }^{36)}$ Prostaglandin E2 is another immunoregulatory molecule strongly upregulated in 3D MSCs. ${ }^{37)}$ It has been reported that 3D culture of UC-MSCs yields a higher immunosuppressive ability but also a less immunogenic phenotype. ${ }^{11)}$
After three-dimensional culture, MSCs were also changing their metabolism preferences and cell communication. ${ }^{38)}$

Preservation of MSC stemness is one of the clinical significant aspects of MSCs. The 3D MSC had higher stemness and differentiation potentials compared with the 2D monolayer MSC culture. ${ }^{39-44)}$ Another interesting feature is that 3D prolongs replicative lifespan or delays cell senescence of MSCs, with increased gene expression of pluripotency marker genes (NANOG, SOX2, and POU5F1/OCT4). ${ }^{45}$ ) This concept is supported by colony formation assays, which measure the proportion of early progenitors in culture. ${ }^{46)}$ Oxygen reaches the inside of three-dimensional structure through diffusion, which makes the internal core of structure hypoxic. ${ }^{47)}$ Hypoxia inducible factor is a master transcription factor that regulates expression of hypoxia-associated genes, which have a important role in MSC self-renewal and production of growth factors and cytokines in hypoxia. ${ }^{48)}$ In addition, 3D MSCs were shown to acquire epigenetic changes. ${ }^{49)}$

With respect to 3D culture technology for MSCs, spherical culture is the most common method at present. In addition, polymer scaffold and perfusion-based 3D culture systems are used. ${ }^{35,50-53)}$ MSCs can maintain the structural basis of cell-cell and cell-matrix contact by aggregation, thereby preventing cell loss due to apoptosis and improving implantation into host tissues. ${ }^{54)}$ Our experimental results show that MSCs in the 3D group express higher levels of ECM factors (e.g., CoL-I, fibronectin, and integrin), and more effectively resist IFN- $\gamma$-induced apoptosis. Other studies have also confirmed that $3 \mathrm{D}$ culture can promote ECM expression and angiogenic cytokine production, such as vascular endothelial growth factor, hepatocyte growth factor, fibroblast growth factor 2, and ANGPT2. ${ }^{35,55)}$ Thus, these angiogenic factors promote the differentiation of MSCs into endothelial progenitor cells (EPC) and endothelial cells. Our results show that MSCs cultured in 3D express more CD31 and CD34, two main surface antigens of early EPCs. ${ }^{56)}$ This indicates that some MSCs have undergone endothelial differentiation. Another noteworthy phenomenon is the expression rate of $\mathrm{CD} 105$, one criterion for identifying MSCs. ${ }^{57)}$ In this study, we demonstrated that CD105 expression decreased after 3D culture, which needs further study.

Based on these results, 3D cultured MSCs administered by intravenous injection can promote the suppressive cytokines IL-10, inhibit the inflammatory factor IFN- $\gamma$, and effectively increase the number of platelets in the peripheral blood of ITP model mice. In the future, the minimum effective dose of MSCs by 3D culture should be further determined. Advanced methods for separating and harvesting MSCs from 3D scaffolds have been developed. ${ }^{58)}$ Moreover, the effects of the material, porosity, and hardness of scaffolds on MSCs require further study. ${ }^{59)}$

In conclusion, 3D MSCs can restore the number of platelets by upregulating immune regulation and ECM expression and therefore have promising therapeutic efficacy in ITP. The 3D culture of MSCs can be considered an upgrade for the treatment of ITP compared with established approaches. However, further studies of safety and efficacy in large animal ITP models are needed.

Acknowledgments We thank Dr. Li Anna, from the Shandong maternity hospital, for providing us with flow anal- 
ysis technology support.

This work was supported by Department of Science \& Technology of Shandong Province (ZR2018MH012) and Quancheng Industrial Leader Project (2017018); and Ji'nan Science and Technology Development Foundation (201704066).

Author Contributions Xiangcui Gong is responsible for cytological experiments and manuscript writing. Di Sun is responsible for gene expression analysis. Zhenghao Li is responsible for image analysis. Qing Shi and Dong Li are responsible for animal experiment. Xiuli $\mathrm{Ju}$ is responsible for the overall experimental design and data analysis.

Conflict of Interest The authors declare no conflict of interest.

Supplementary Materials The online version of this article contains supplementary materials.

\section{REFERENCES}

1) Audia S, Mahévas M, Samson M, Godeau B, Bonnotte B. Pathogenesis of immune thrombocytopenia. Autoimmun. Rev., 16, 620-632 (2017).

2) Pérez-Simón JA, Tabera S, Sarasquete ME, Díez-Campelo M, Canchado J, Sánchez-Abarca LI, Blanco B, Alberca I, Herrero-Sánchez C, Cañizo C, San Miguel JF. Mesenchymal stem cells are functionally abnormal in patients with immune thrombocytopenic purpura. Cytotherapy, 11, 698-705 (2009).

3) Stasi R. Immune thrombocytopenia: pathophysiologic and clinical update. Semin. Thromb. Hemost., 38, 454-462 (2012).

4) Zhao X, Ding HF, Guo CS, Lu XJ, Xu M, Xing J, Han F, Wang $\mathrm{L}$, Lu G, Liu GQ. In vitro effects of mesenchymal stem cells on secreting function of $\mathrm{T}$ lymphocytes and $\mathrm{CD} 4^{+} \mathrm{CD} 25^{+} \mathrm{T}$ cells from patients with immune thrombocytopenia. Zhonghua Xue Ye Xue $\mathrm{Za}$ Zhi, 34, 1015-1019 (2013).

5) Wang $X$, Yin $X$, Sun W, Bai J, Shen Y, Ao Q, Gu Y, Liu Y. Intravenous infusion umbilical cord-derived mesenchymal stem cell in primary immune thrombocytopenia: a two-year follow-up. Exp. Ther. Med., 13, 2255-2258 (2017).

6) Wu M, Ge H, Li S, Chu H, Yang S, Sun X, Zhou Z, Zhu X. Mesenchymal stem cells immunosuppressed IL-22 in patients with immune thrombocytopenia via soluble cellular factors. J. Immunol. Res., 2015, 316351 (2015).

7) Wu Z, Zhang S, Zhou L, Cai J, Tan J, Gao X, Zeng Z, Li D. Thromboembolism induced by umbilical cord mesenchymal stem cell infusion: a report of two cases and literature review. Transplant. Proc., 49, 1656-1658 (2017).

8) Zhou Y, Chen $\mathrm{H}, \mathrm{Li} \mathrm{H}, \mathrm{Wu}$ Y. 3D culture increases pluripotent gene expression in mesenchymal stem cells through relaxation of cytoskeleton tension. J. Cell. Mol. Med., 21, 1073-1084 (2017).

9) Cosgrove BD, Mui KL, Driscoll TP, Caliari SR, Mehta KD, Assoian RK, Burdick JA, Mauck RL. $N$-Cadherin adhesive interactions modulate matrix mechanosensing and fate commitment of mesenchymal stem cells. Nat. Mater., 15, 1297-1306 (2016).

10) Qazi TH, Mooney DJ, Duda GN, Geissler S. Biomaterials that promote cell-cell interactions enhance the paracrine function of MSCs. Biomaterials, 140, 103-114 (2017).

11) Li Y, Wu Q, Wang Y, Li L, Chen F, Shi Y, Bu H, Bao J. Immunogenicity of hepatic differentiated human umbilical cord mesenchymal stem cells promoted by porcine decellularized liver scaffolds. Xenotransplantation, 24, e12287 (2017).

12) Xu X, Li D, Li X, Shi Q, Ju X. Mesenchymal stem cell conditioned medium alleviates oxidative stress injury induced by hydrogen per- oxide via regulating miR143 and its target protein in hepatocytes. BMC Immunol., 18, 51 (2017).

13) Qi L, Wang R, Shi Q, Yuan M, Jin M, Li D. Umbilical cord mesenchymal stem cell conditioned medium restored the expression of collagen II and aggrecan in nucleus pulposus mesenchymal stem cells exposed to high glucose. J. Bone Miner. Metab., 37, 455-466 (2019).

14) Lotfy A, Salama M, Zahran F, Jones E, Badawy A, Sobh M. Characterization of mesenchymal stem cells derived from rat bone marrow and adipose tissue: a comparative study. Int. J. Stem Cells, 7 , 135-142 (2014).

15) Cines DB, Bussel JB, Liebman HA, Luning Prak ET. The ITP syndrome: pathogenic and clinical diversity. Blood, 113, 6511-6521 (2009).

16) Katsman $\mathrm{Y}$, Foo AH, Leontyev D, Branch DR. Improved mouse models for the study of treatment modalities for immune-mediated platelet destruction. Transfusion, 50, 1285-1294 (2010).

17) Neschadim A, Branch DR. Mouse models of autoimmune diseases: immune thrombocytopenia. Curr. Pharm. Des., 21, 2487-2497 (2015).

18) Rodrigues CE, Capcha JM, de Bragança AC, Sanches TR, Gouveia PQ, de Oliveira PA, Malheiros DM, Volpini RA, Santinho MA, Santana BA, Calado RD, Noronha IL, Andrade L. Human umbilical cord-derived mesenchymal stromal cells protect against premature renal senescence resulting from oxidative stress in rats with acute kidney injury. Stem Cell Res. Ther., 8, 19 (2017).

19) Zhang W, Wang Y, Kong J, Dong M, Duan H, Chen S. Therapeutic efficacy of neural stem cells originating from umbilical cordderived mesenchymal stem cells in diabetic retinopathy. Sci. Rep., 7, 408 (2017).

20) Oppliger B, Joerger-Messerli M, Mueller M, Reinhart U, Schneider $\mathrm{P}$, Surbek DV, Schoeberlein A. Intranasal delivery of umbilical cord-derived mesenchymal stem cells preserves myelination in perinatal brain damage. Stem Cells Dev., 25, 1234-1242 (2016).

21) Zou X, Gu D, Xing X, Cheng Z, Gong D, Zhang G, Zhu Y. Human mesenchymal stromal cell-derived extracellular vesicles alleviate renal ischemic reperfusion injury and enhance angiogenesis in rats. Am. J. Transl. Res., 8, 4289-4299 (2016).

22) Romieu-Mourez R, Coutu DL, Galipeau J. The immune plasticity of mesenchymal stromal cells from mice and men: concordances and discrepancies. Front. Biosci. (Elite Ed.), 4, 824-837 (2012), Elite Ed.

23) Meisel R, Zibert A, Laryea M, Göbel U, Däubener W, Dilloo D. Human bone marrow stromal cells inhibit allogeneic T-cell responses by indoleamine 2,3-dioxygenase-mediated tryptophan degradation. Blood, 103, 4619-4621 (2004).

24) Ren G, Su J, Zhang L, Zhao X, Ling W, L'huillie A, Zhang J, Lu Y, Roberts AI, Ji W, Zhang H, Rabson AB, Shi Y. Species variation in the mechanisms of mesenchymal stem cell-mediated immunosuppression. Stem Cells, 27, 1954-1962 (2009).

25) Chinnadurai R, Ng S, Velu V, Galipeau J. Challenges in animal modelling of mesenchymal stromal cell therapy for inflammatory bowel disease. World J. Gastroenterol., 21, 4779-4787 (2015).

26) Augello A, Tasso R, Negrini SM, Amateis A, Indiveri F, Cancedda R, Pennesi G. Bone marrow mesenchymal progenitor cells inhibit lymphocyte proliferation by activation of the programmed death 1 pathway. Eur. J. Immunol., 35, 1482-1490 (2005).

27) Gu YZ, Xue Q, Chen YJ, Yu GH, Qing MD, Shen Y, Wang MY, Shi Q, Zhang XG. Different roles of PD-L1 and FasL in immunomodulation mediated by human placenta-derived mesenchymal stem cells. Hum. Immunol., 74, 267-276 (2013).

28) Quaedackers ME, Baan CC, Weimar W, Hoogduijn MJ. Cell contact interaction between adipose-derived stromal cells and allo-activated T lymphocytes. Eur. J. Immunol., 39, 3436-3446 (2009).

29) Di Nicola M, Carlo-Stella C, Magni M, Milanesi M, Longoni PD, Matteucci P, Grisanti S, Gianni AM. Human bone marrow stromal 
cells suppress T-lymphocyte proliferation induced by cellular or nonspecific mitogenic stimuli. Blood, 99, 3838-3843 (2002).

30) Aggarwal S, Pittenger MF. Human mesenchymal stem cells modulate allogeneic immune cell responses. Blood, 105, 1815-1822 (2005).

31) Engela AU, Hoogduijn MJ, Boer K, Litjens NH, Betjes MG, Weimar W, Baan CC. Human adipose-tissue derived mesenchymal stem cells induce functional de-novo regulatory $\mathrm{T}$ cells with methylated FOXP3 gene DNA. Clin. Exp. Immunol., 173, 343-354 (2013).

32) Maggini J, Mirkin G, Bognanni I, Holmberg J, Piazzón IM, Nepomnaschy I, Costa H, Cañones C, Raiden S, Vermeulen M, Geffner JR. Mouse bone marrow-derived mesenchymal stromal cells turn activated macrophages into a regulatory-like profile. PLoS ONE, 5, e9252 (2010).

33) Ren G, Zhang L, Zhao X, Xu G, Zhang Y, Roberts AI, Zhao RC, Shi Y. Mesenchymal stem cell-mediated immunosuppression occurs via concerted action of chemokines and nitric oxide. Cell Stem Cell, 2, 141-150 (2008).

34) Klyushnenkova E, Mosca JD, Zernetkina V, Majumdar MK, Beggs KJ, Simonetti DW, Deans RJ, McIntosh KR. T cell responses to allogeneic human mesenchymal stem cells: immunogenicity, tolerance, and suppression. J. Biomed. Sci., 12, 47-57 (2005).

35) Kim S, Han YS, Lee JH, Lee SH. Combination of MSC spheroids wrapped within autologous composite sheet dually protects against immune rejection and enhances stem cell transplantation efficacy. Tissue Cell, 53, 93-103 (2018).

36) Bartosh TJ, Ylöstalo JH, Mohammadipoor A, Bazhanov N, Coble K, Claypool K, Lee RH, Choi H, Prockop DJ. Aggregation of human mesenchymal stromal cells (MSCs) into 3D spheroids enhances their antiinflammatory properties. Proc. Natl. Acad. Sci. U.S.A., 107, 13724-13729 (2010).

37) Ylöstalo JH, Bartosh TJ, Coble K, Prockop DJ. Human mesenchymal stem/stromal cells cultured as spheroids are self-activated to produce prostaglandin E2 that directs stimulated macrophages into an anti-inflammatory phenotype. Stem Cells, 30, 2283-2296 (2012).

38) Bartosh TJ, Ylostalo JH. Efficacy of 3D culture priming is maintained in human mesenchymal stem cells after extensive expansion of the cells. Cells, 8, 1031 (2019).

39) Imamura A, Kajiya H, Fujisaki S, Maeshiba M, Yanagi T, Kojima $\mathrm{H}$, Ohno J. Three-dimensional spheroids of mesenchymal stem/stromal cells promote osteogenesis by activating stemness and Wnt $/ \beta$ catenin. Biochem. Biophys. Res. Commun., 523, 458-464 (2020).

40) Frith JE, Thomson B, Genever PG. Dynamic three-dimensional culture methods enhance mesenchymal stem cell properties and increase therapeutic potential. Tissue Eng. Part C Methods, 16, 735-749 (2010)

41) Wang W, Itaka K, Ohba S, Nishiyama N, Chung UI, Yamasaki Y, Kataoka K. 3D spheroid culture system on micropatterned substrates for improved differentiation efficiency of multipotent mesenchymal stem cells. Biomaterials, 30, 2705-2715 (2009).

42) Cheng NC, Wang S, Young TH. The influence of spheroid formation of human adipose-derived stem cells on chitosan films on stemness and differentiation capabilities. Biomaterials, 33, 1748-1758 (2012).

43) Arufe MC, De la Fuente A, Fuentes-Boquete I, De Toro FJ, Blanco FJ. Differentiation of synovial CD-105(+) human mesenchymal stem cells into chondrocyte-like cells through spheroid formation. $J$. Cell. Biochem., 108, 145-155 (2009).

44) Miyagawa Y, Okita H, Hiroyama M, Sakamoto R, Kobayashi M, Nakajima H, Katagiri YU, Fujimoto J, Hata J, Umezawa A, Kiyokawa N. A microfabricated scaffold induces the spheroid formation of human bone marrow-derived mesenchymal progenitor cells and promotes efficient adipogenic differentiation. Tissue Eng. Part A, 17, 513-521 (2011).

45) Cheng NC, Chen SY, Li JR, Young TH. Short-term spheroid formation enhances the regenerative capacity of adipose-derived stem cells by promoting stemness, angiogenesis, and chemotaxis. Stem Cells Transl. Med., 2, 584-594 (2013).

46) Pochampally R. Colony forming unit assays for MSCs. Methods Mol. Biol., 449, 83-91 (2008).

47) Sart S, Tsai AC, Li Y, Ma T. Three-dimensional aggregates of mesenchymal stem cells: cellular mechanisms, biological properties, and applications. Tissue Eng. Part B Rev., 20, 365-380 (2014).

48) Tamama K, Kawasaki H, Kerpedjieva SS, Guan J, Ganju RK, Sen CK. Differential roles of hypoxia inducible factor subunits in multipotential stromal cells under hypoxic condition. J. Cell. Biochem., 112, 804-817 (2011).

49) Nishida H, Suzuki T, Kondo S, Miura H, Fujimura Y, Hayashizaki Y. Histone $\mathrm{H} 3$ acetylated at lysine 9 in promoter is associated with low nucleosome density in the vicinity of transcription start site in human cell. Chromosome Res., 14, 203-211 (2006).

50) Redondo-Castro E, Cunningham CJ, Miller J, Brown H, Allan SM, Pinteaux E. Changes in the secretome of tri-dimensional spheroidcultured human mesenchymal stem cells in vitro by interleukin-1 priming. Stem Cell Res. Ther., 9, 11 (2018).

51) Yan L, Jiang B, Li E, Wang X, Ling Q, Zheng D, Park JW, Chen X, Cheung E, Du X, Li Y, Cheng G, He E, Xu RH. Scalable generation of mesenchymal stem cells from human embryonic stem cells in 3D. Int. J. Biol. Sci., 14, 1196-1210 (2018).

52) Caires HR, Barros da Silva P, Barbosa MA, Almeida CR. A coculture system with three different primary human cell populations reveals that biomaterials and MSC modulate macrophage-driven fibroblast recruitment. J. Tissue Eng. Regen. Med., 12, e1433-e1440 (2018).

53) Seifert M, Lubitz A, Trommer J, Könnig D, Korus G, Marx U, Volk HD, Duda G, Kasper G, Lehmann K, Stolk M, Giese C. Crosstalk between immune cells and mesenchymal stromal cells in a 3D bioreactor system. Int. J. Artif. Organs, 35, 986-995 (2012).

54) Lee EJ, Park SJ, Kang SK, Kim GH, Kang HJ, Lee SW, Jeon HB, Kim HS. Spherical bullet formation via E-cadherin promotes therapeutic potency of mesenchymal stem cells derived from human umbilical cord blood for myocardial infarction. Mol. Ther., 20, 1424-1433 (2012)

55) Potapova IA, Brink PR, Cohen IS, Doronin SV. Culturing of human mesenchymal stem cells as three-dimensional aggregates induces functional expression of CXCR4 that regulates adhesion to endothelial cells. J. Biol. Chem., 283, 13100-13107 (2008).

56) Cheng CC, Chang SJ, Chueh YN, Huang TS, Huang PH, Cheng SM, Tsai TN, Chen JW, Wang HW. Distinct angiogenesis roles and surface markers of early and late endothelial progenitor cells revealed by functional group analyses. BMC Genomics, 14, 182 (2013)

57) Wang D, Liu N, Xie Y, Song B, Kong S, Sun X. Different culture method changing CD105 expression in amniotic fluid MSCs without affecting differentiation ability or immune function. J. Cell. Mol. Med., 24, 4212-4222 (2020).

58) Lam AT, Sim EJ, Shekaran A, Li J, Teo KL, Goggi JL, Reuveny S, Birch WR, Oh SK. Sub-confluent culture of human mesenchymal stromal cells on biodegradable polycaprolactone microcarriers enhances bone healing of rat calvarial defect. Cytotherapy, 21, 631-642 (2019).

59) Li H, Shen S, Fu H, Wang Z, Li X, Sui X, Yuan M, Liu S, Wang G, Guo Q. Immunomodulatory functions of mesenchymal stem cells in tissue engineering. Stem Cells Int., 2019, 9671206 (2019). 